\title{
MESOPLODON DENSIROSTRIS (CETACEA, ZIPHIDAE), PRIMEIRO REGISTRO PARA O ATLÁNTICO SUL OCIDENTAL *
}

\author{
HUGO P. CASTELLO Q MARIA CRISTINA PINEDO \\ Base Ocesnográfica Atlântica \\ Fundaçăo Universidade de Rio Grande, Rio Grande, RS, Brasil
}

\section{SYNOPSIS}

A female Blainville's beaked whale, Mesoplodon densirostris, $4.4 \mathrm{~m}$ long was found stranded at $42 \mathrm{~km}$, South of Cassino, Rio Grande do Sul, Brazil. The triangular shape and dimensions of the pair of unerupted teeth $(60 \times 45 \times 11 \mathrm{~mm})$ in the lower jaw, together with the distance $(180 \mathrm{~mm})$ from the alveoli to the hinder edge of the mandibular symphysis proved to be a good systematic character for females of this species. The vertebral formula is $C 7+D 10+L 11+C a 15(+2)=45$. It presents 10 pairs of ribs, 6 being double headed. M. densirostris is the only species of Mesoplodon which seems normally to occur both north and south of the equator and also the onlv species of this genus to strand on oceanic island of tropical and warm-temperature waters. The present record is the first for the South West Attantic waters.

Introdução

Em 5 de maio de 1977, uma fêmea de "golfinho bicudo", de $4.4 \mathrm{~m}$ de comprimento foi achada encalhada na praia a $42 \mathrm{~km}$ ao sul do Cassino, RS. Quando foi examinada pela primeira vez, já havia transcorrido aproximadamente uma semana desde a morte do mesmo. Obtiveram-se algumas medidas (Tab. I) e slides coloridos. O cadaver foi carneado na praia com a ajuda dos técnicos do Museu Oceanográfico de Rio Grande e o esqueleto foi recuperado (MORG 062).

\begin{tabular}{|c|c|c|c|c|c|c|c|c|c|c|}
\hline \multirow[t]{2}{*}{ Tabela I - Medidas corporais de $M$. densirostris } & \multirow{2}{*}{$\begin{array}{l}\text { MORG } \\
\mathrm{cm}\end{array}$} & \multirow{2}{*}{$\begin{array}{l}062 \\
\%\end{array}$} & \multicolumn{2}{|c|}{$\begin{array}{c}\text { TK } 256 \\
\text { Ocean.Res.Inst. }\end{array}$} & \multicolumn{2}{|c|}{ Allen (1906) } & \multicolumn{2}{|c|}{ Gioller (1966) } & \multicolumn{2}{|c|}{ Raven (1942) } \\
\hline & & & $\mathrm{cm}$ & $\%$ & $\mathrm{~cm}$ & $\%$ & $\mathrm{~cm}$ & $\%$ & $\mathrm{~cm}$ & $\%$ \\
\hline $\begin{array}{l}\text { 01. Comprimento total, do extremo mand. sup. } \\
\text { até reentrância caudal. } \\
\text { 02. Comprimento do extremo mand. sup. até res- } \\
\text { piradouro. } \\
\text { 03. Comprimento do extremo mand-sup. até cen- } \\
\text { tro do olho. } \\
\text { 04. Comprimento do extremo mand.sup. até a } \\
\text { comissura bucal. } \\
\text { 05. Comprimento do extremo mand.sup. até } \\
\text { inserção ant. da peitoral. } \\
\text { 06. Comprimento do centro do olho até a comissu- } \\
\text { ra bucal. } \\
\text { 07. Comprimento do centro do olho até o respira- } \\
\text { douro. } \\
\text { 08. Comprimento do extremo mand.sup. até extre- } \\
\text { mo da dorsal. } \\
\text { 09. Comprimento do sulco da garganta. } \\
\text { 10. Comprimento do extremo mand.sup. até o extre- } \\
\text { mo anterior do sulco da garganta. } \\
\text { 11. Altura da abertura do olho. } \\
\text { 12. Comprimento da abertura do olho. } \\
\text { 13. Comprimento do respiradouro. } \\
\text { 14. Largura do respiradouro. } \\
\text { 15. Comprimento da peitoral, da inserção anterior } \\
\text { até o extremo. } \\
\text { 16. Comprimento da peitoral, da axila até o } \\
\text { extremo. } \\
\text { 17. Largura máxima da peitoral. } \\
\text { 18. Altura da nadadeira dorsal. } \\
\text { 19. Comprimento da base da dorsal. } \\
\text { 20. Largura da caudal. } \\
\text { 21. Comp. da reent. caudal até a ins. ant. da cauda. } \\
\text { 22. Profundidade da reentrância caudal. } \\
\text { 23. Perímetro maximo do corpo. }\end{array}$ & $\begin{array}{c}2,5 \\
2,7 \\
3,3 \\
7,5 \\
\\
41,2 \\
\\
32,5 \\
12,5 \\
25 \\
35 \\
75 \\
27,5 \\
1,2 \\
142\end{array}$ & $\begin{array}{r}0,5 \\
0,6 \\
0,7 \\
1,7 \\
9,2 \\
7,2 \\
2,8 \\
5,6 \\
7,8 \\
16,8 \\
0,2 \\
31,0\end{array}$ & $\begin{array}{l}41 \\
30 \\
12 \\
19 \\
28 \\
80 \\
28\end{array}$ & $\begin{array}{r}11,5 \\
\\
8,4 \\
3,4 \\
5,3 \\
7,9 \\
22,5 \\
7,9\end{array}$ & $\begin{array}{l}- \\
-\end{array}$ & $\begin{array}{c}100 \\
- \\
- \\
8,5 \\
-\end{array}$ & $\begin{array}{l}- \\
- \\
-\end{array}$ & $\begin{array}{c}100 \\
- \\
- \\
-\end{array}$ & - & $\begin{array}{c}13,9 \\
- \\
23,1\end{array}$ \\
\hline
\end{tabular}

Quando o cránio e as mandíbulas foram fervidos para a limpeza, observou-se que o par de dentes da mandíbula inferior não haviam eclodido, o que é uma característica distintiva das fêmeas. $\mathrm{O}$ dente direito foi extraído, medido e fotografado (Fig. 3). Como existiam diferenças com os dentes de Mesoplodon grayi, M. layardi e $M$. hectori, as únicas espécies registradas até agora no Atlântico Sul Ocidental, medidas e fotografias do dente foram enviadas aos Dr. McCann (Nova Zelândia) e Dr. Joseph Moore (USA). Ambos especialistas nos ajuaaram na determinação deste exemplar de $M e s o$. plodon densirostris, neste trabalho, mencionado pela primeira vez para as águas do Attântico Sul Ocidental. Para ambos especialistas nossos sinceros agradecimentos extensivo ao pessoal do Museu Oceanográfico.
Morfologia

Quando examinado na praia o cadaver estava repleto de gases e danificado. A primeira vista a coloração externa parecia ser escura, dependendo da direção da luz solar. Contudo, ao observar os slides coloridos nota-se uma tonalidade azulaḍa no corpo. Não foi observada uma área esbranquiçada na regiâo ventral, provavelmente devido à adesão de areia no tegumento. Também não apresentava marcas ou cicatrizes na pele, tão comuns no gênero Mesoplodon (McCann, 1974).

As medicões externas do exemplar (MORG 062) foram comparad as (Tab. I) com as ae outros especmens estudados por especialistas no Hemisfério Norte (Kasuya \& Nishiwaki, 1971). 
Os cetáceos desta espécie, também conhecida vulgarmente como "golfinho bicudo de Blainville" atingem até $5,2 \mathrm{~m}$ de comprimento, sendo nosso exemplar de $4,4 \mathrm{~m}$, a maior fêmea até o presente registrada. $\mathrm{O}$ corpo tem forma alongada. A cabeça, cujo con- torno é uma das caracteristicas distintivas da espécie, está majs marcada nos machos que nas fêmeas. Nos machos existe uma elevaçăo proeminente perto da comissura bucal, de cada lado da boca (Fig.1A) e pode observar-se que a cúspide do dente emerge do lábio.

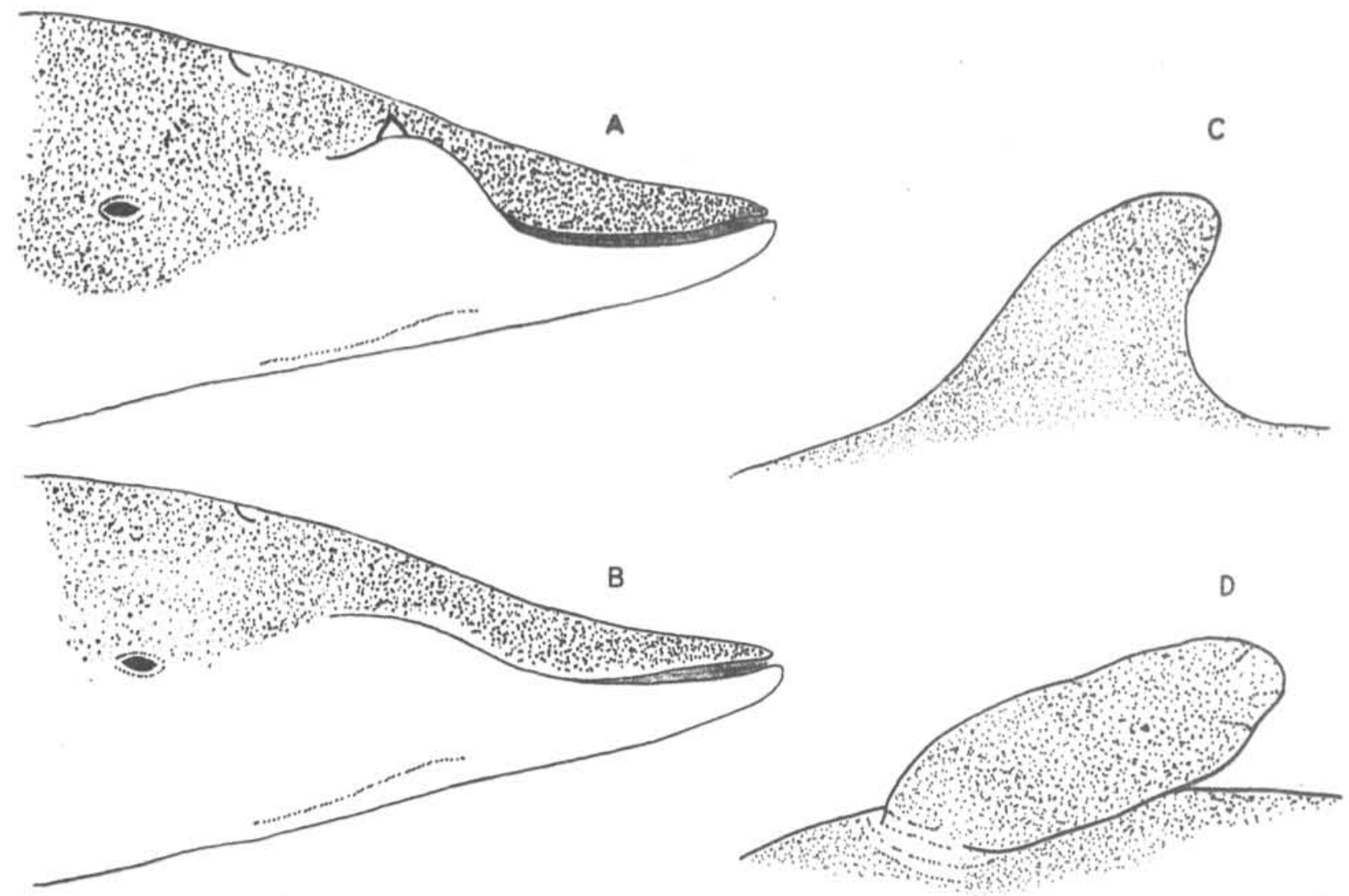

Fig. 1 - Mesoplodon densirostris - A-macho (em base a uma fotografia publicada por Leatherwood et al. (1976). B-fêmea MORG 062, C-nadadeira dorsal, D-nadadeira peitoral esquerda (B,C,D em base a slides).

Nas fêmeas o dente não eclode e a mandíbula não apresenta a elevação proeminente.

\section{Nadadeiras}

A nadadeira dorsal, localizada atrás do ponto médio do dorso é proeminente, mas parcialmente destruída em nosso espécimen (Fig. 1C). As peitorais são alongadas $(9,2 \%$ do comprimento total) e tem forma ovalada (Fig. 1D). A nadadeira caudal representa
$16,8 \%$ do comprimento. Não existe marca alguma de reentrâncıa mediana na cauda.

\section{Crânio}

Apresenta um comprimento côndilo-basal de $770 \mathrm{~mm}$ (Tab. II). A metade posterior da mandíbula possui um alargamento visível (Fig. 2A, B) nos machos, e muito menos acentuado nas - fêmeas (Fig. 2C,D).
$\mathbf{A}$

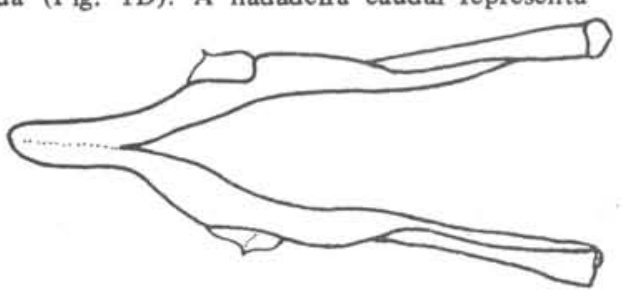

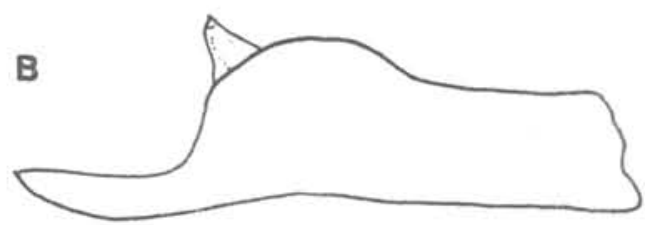

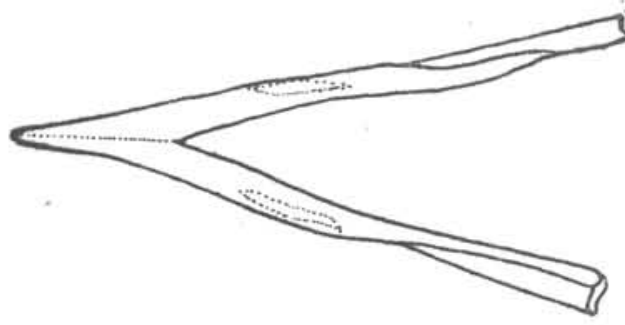

D

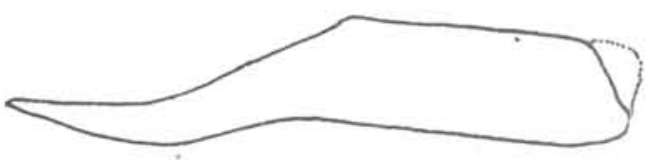

Fig. 2 - Esquemas das mandíbulas, baseados em fotos de $M$. densirostris $(A, B)$ e $(C, D)$ publicadas por Raven (1942). 
Tabela II - Medidas do crânio (milímetros) de Mesoplodon densirostris do Oceano Atlântico Sul Ocidental

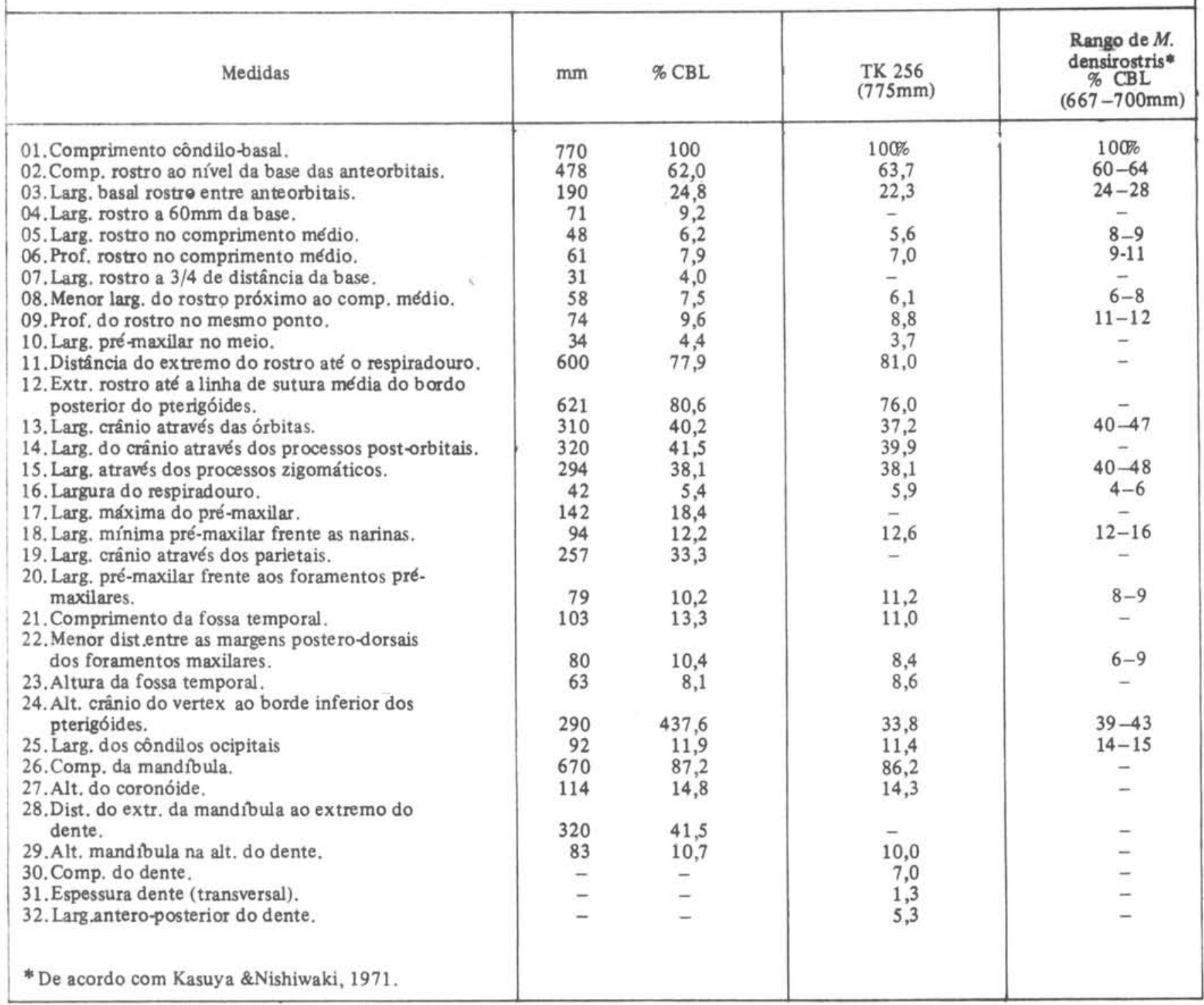

$\mathrm{O}$ dente da mandibula inferior nâo eclode, mas quando foi extraido apresentava a cavidade polpar completamente vedada, tem forma triangular, lateralmente comprimido e ligeiramente curva para trás (Fig. 3). Suas dimensōes são: $60 \mathrm{~mm}$ x $45 \mathrm{~mm} \times 11 \mathrm{~mm}$. $O$ dentículo presente no extremo é pontiagudo e sem esmal te; a base é irregular com várias raízes pontudas. $O$ dente está localizado bem atrás do bordo posterior da sínfisis mandibular $(180 \mathrm{~mm})$ e sobre a plataforma da mandibula. $O$ dentículo de dente está $320 \mathrm{~mm}$ do ex tremo anterior da mandibula inferior.

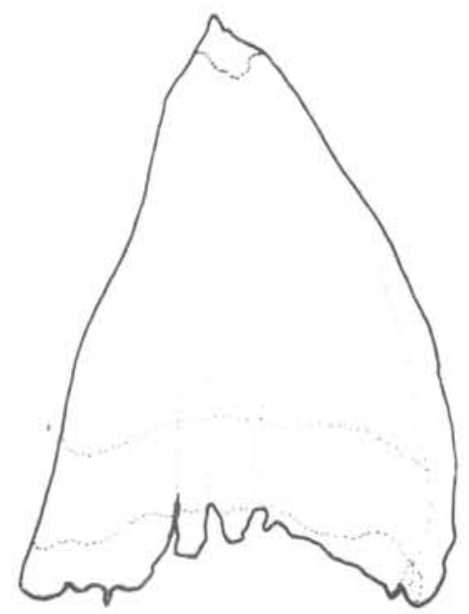

Fig. 3 - Dente da fêmea MORG 062 de $M$. densirostris.
Várias medidas importantes do crânio aparecem na Tabela 2, comparadas com as do exemplar TK 256 do Japão e com a rango de variação para a espécie, segundo Kasuya-Nishiwaki (op. cit.).

Ambos foramens pré-maxilares encontram-se em linha e a 17 $\mathrm{mm}$ em linha oblíqua da margem postero-dorsal do foramen maxilar. O sulco brasirostral está mal definido.

\section{Esqueleto post-craneal}

O total das vértebras achadas foi de 43 , mas nota-se que faltam duas caudais (43.a 44 a) ou seja, o total é 45 , com a seguinte fórmula: C7 + D10 + L11 + Ca15 (+2) $=45$.

Apresenta 10 pares de costelas, 6 das quais tém dupla cabeça.

\section{Distribuição geográfica}

O golfinho bicudo de Blainville, $M$. densirostris é uma espécie rara de Odontoceto, com 20 registros conhecidos para o Pacífico Norte, Pacífico Sul, Indico e Attântico Norte (Kasuya \& Nishiwaki, op. cit.) Mais recentemente Ross coletou nove exemplares mais nas costas da África do Sul sobre o Oceano Índico.

$\mathrm{O}$ presente registro corroboraria mais ainda com a afirmação feita por Davies (1963) de que Mesoplodon densirostris é a única espécie de Mesoplodon que parece estar presente tanto ao Norte como ao Sul da linha do Equador. E também a única espécie que encalha em ilhas oceânicas de marés tropicais ou temperado-cálidas. De acordo com Moore (1966) a população vivente de densirostris tem uma distribuição muito mais mar afora que qualquer outra espécie do A tlântico e muito mais austral (no Hemisfério Norte) que outras, exceto $M$, europaeus. 
Até o presente momento, dois golfinhos bicudos têm sido registrados em águas brasileiras. Carvalho (1969) informou sobre um golfinho bicudo de Cuvier, Ziphius cavirostris, encalhado perto de São Paulo e maiı recentemente Gianuca \& Castello (1976) informaram sobre o encalhe de um exemplar de "golfinho bicudo focinho de garrafa", Hyperoodon planifrons, na costa do Rio Grande do Sul.

Mais ao sul, no Uruguay, de acordo com Praderi (1972) há um registro de Mesoplodon layardi. Para a Argentina, M. grayi é conhecida em base a dois registros de Pcia. Buenos Aires: o primeiro trata-se de um crânio coletado em Quequén e descrito por De Carli \& Aramayo (1970) e o segundo em Monte Hermoso e descrito por De Carli e colaboradores.

Com a exceção do registro de $Z$. cavirostris de São Paulo, entre os $20^{\circ}$ e $30^{\circ}$ de Latitude Sul conheciam-se três golfinhos pertencentes à famılia Ziphiidae ou seja, Hyperoodon planifrons, M. layardi e $M$. grayi . Deve-se agora então agregar $M$. densirostris, que representa um gênero e espécie não citados para a fauna marinha brasileira.

\section{Bibiiografia}

CARVALHO, J. P. 1969. Sobre a provável ocorrência de "Baleia de Cuvier" no litoral de São Paulo. Rev. nac. Pesca, 10(82):8-11.

DAVIES, J.L. 1963. The antitropical factor in Cetacean speciation. Evolution, 17(1):107-116

DE CARLI, M.J. URQUIOLA DE \& ARAMAYO, S.A. 1970. Descripción de um Mesoplodon sp. hallado en la localidad de Quequén, Pcia. de Buenos Aires. Neotropica, 16(51):107-118, fig. 5.
DE CARLI, M.J. URQUIOLA DE, ARAMAYO, S.A.\& PINERO, M Sobre el hallazgo de Mesoplodon grayi en la playa de Monte Hermoso, Pcia. de Buenos Aires. An. Com. Inv. Cient. Pcia. de Buenos Aires, La Plata (manuscrito).

GIANUCA, N.M. \& CASTELLO H.P. First record of the southern bottle-nose whale, Hyperoodon planifrons, from Rio Grande do Sul, Brazil (Cetacea, Ziphiidae). Sci. Rep. Whales Res. Inst. Japan, (29): $107-111$.

KASUYA, T. \& NISHIWAKI M. 1971. First record of Mesoplodon densirostris from Formosa. Sci. Rep. Whales Res. Inst. Japan, (23):129-137.

LEATHERWOOD, S.; CALDWELL, D.K. \& WINN, H.E. 1976. Whales, dolphins and porpoises of the Western North Atlan. tic. A guide to their identification. N.O.A.A. Tech. Rep. N.M.F.S. Circ. (396):1-173.

MoCANN, CH. 1974. Body scarring on cetacean odontocets. Sci. Rep. Whales Res. Inst. Japan, (26):145-155.

MOORE, J.C. 1966. Diagnoses and distributions of beaked whales of the genus Mesoplodon known from North American waters. In: Norris, K., ed. - Whales, dolphins and porpaises. Berkeley, Univ. of California Press, 789 p.

PRADERI, R. 1972. Notas sobre un ejemplar de Mesoplodon layar. di (Gray) (Cetacea, Hyperoodontidae) de la costa Atlantica del Uruguay. Com Zool. Mus. Hist. Nat. Montevideo, 10(137): 1-7.

RAVEN, H.C. 1942. On the structure of Mesoplodon densirostris, a rare beaked whale. Bull. Amer. Mus. Nat. Hist., $80: 23-50$.

ROSS, G.J.B. Chapter about Mesoplodon densirostris. (Tese não publicada). 